\title{
¿ENERGÍA NUCLEAR EN CHILE?* \\ LOS COSTOS Y BENEFICIOS DE LA OPCIÓN DE CONSTRUIR UNA CENTRAL NUCLEAR EN 2020
}

\section{Geoffrey Rothwell}

En este trabajo se evalúa el costo de la opción de ser capaz de comenzar a construir la primera central nuclear en Chile en 2020. Lo anterior implica que para esa fecha (1) existe en Chile un regulador competente que cuenta con los conocimientos técnicos necesarios para dar un permiso de operación, que está en condiciones de regular las operaciones de una central nuclear y el manejo del combustible nuclear y que habrá autorizado el lugar y dado un permiso de obras; (2) se habrá designado a un operador y se habrá obtenido el correspondiente financiamiento, y (3) se habrá especificado un sistema

Geoffrey Rothwell. Senior Lecturer en el Departamento de Economía de la Universidad de Stanford, donde también es director asociado del Programa de Políticas Públicas. Ha publicado extensamente sobre todos los aspectos de la economía de la energía nuclear. También ha participado en numerosos grupos asesores sobre política nuclear tales como el Generation IV Roadmap Committee, el Comité sobre Metodología para Analizar el Desempeño de Plantas Nucleares de la International Atomic Energy Agency (AEA), que presidió, y el Comité de Descontaminación y Descontinuación de Plantas de Enriquecimiento de Uranio.

* Este trabajo fue financiado en parte con un aporte del Centro de Estudios Públicos (CEP), Santiago, Chile. Quisiera agradecer a Julio Vergara, Alexander Galetovic, Rob Graber, Donald Korn, Bruno Philippi, Robert Pindyck, Marcelo Tokman, Bill Rasin, Hugh Rudnick, Kent Williams y a la Sociedad de Fomento Fabril (Sofofa) por sus apreciaciones, referencias, datos, comentarios y palabras de aliento. El trabajo fue presentado en el Simposio Internacional de Aplicación Pacífica de la Tecnología Nuclear en el GCC, Universidad Rey Abdul Aziz, Jeddah, Arabia Saudita, noviembre 3-5, 2008. En este trabajo se reflejan los puntos de vista y las conclusiones del autor, y no las del CEP.

Estudios Públicos, 112 (primavera 2008). 
para el manejo del combustible nuclear y sus desechos. En ese momento el valor actual neto del proyecto podrá calcularse y compararse con otras alternativas. Hasta entonces los fondos deberían gastarse en la elaboración de un marco regulador y en la preparación de la infraestructura física. Según los supuestos y el modelamiento económico de este trabajo, Chile (o cualquier país que contemple la posibilidad de construir su primera central nuclear) debería estar dispuesto a invertir cierto monto en estos preparativos, aun si la central nuclear nunca llegue a construirse. El origen de los beneficios de escoger la opción nuclear es la incertidumbre respecto del establecimiento y la forma que adoptarán los controles internacionales de las emisiones de dióxido de carbono, lo cual podría aumentar enormemente el costo de los combustibles fósiles. Así pues, mientras más incierto sea el costo social o el precio de mercado del dióxido de carbono, mayor será la ventaja implícita de tener la opción de construir centrales nucleares que no emiten carbono. En conclusión, por el momento la inversión más rentable sería poner en marcha una iniciativa permanente para determinar (por ejemplo, a través de sondeos trimestrales) si la opinión pública chilena está dispuesta a comprometer, con conocimiento de causa, su apoyo a un programa nacional de energía nuclear.

Palabras clave: política chilena de energía, economía de la energía nuclear, inversiones bajo incertidumbre, control de emisiones de dióxido de carbono.

Sistema de clasificación: JEL: C15, D81, L94.

Recibido: octubre de 2008. Aceptado: noviembre de 2008.

\section{Nuevas centrales nucleares en economías no nucleares en vías de desarrollo}

\section{A} medida que el cambio climático se transforma en una realidad mundial debido a las emisiones de gases de efecto invernadero tales como el dióxido de carbono $\left(\mathrm{CO}_{2}\right)$, las economías en vías de desarrollo enfrentan un dilema cada vez más complejo porque para crecer deben aumentar su consumo de energía. En particular, necesitan contar con fuentes confiables de energía eléctrica para impulsar los sistemas de tecnología avanzada, pero disponen de pocas alternativas eficaces que no emitan dióxido de carbono. Una alternativa que está siendo evaluada por algunos países es la energía 
nuclear. Antes de analizar si a un país en vías de desarrollo le conviene invertir en energía nuclear, dedicaremos el resto de esta sección a una visión general del ciclo del combustible nuclear y de la industria de la energía nuclear.

\subsection{El ciclo del combustible nuclear}

El uranio natural (U) consta de dos isótopos principales: el 99,3\% es U-238 no radioactivo y el $0,7 \%$ es U-235 radioactivo. A medida que se desintegra, el U-235 libera partículas atómicas que (1) o bien son captadas por elementos no radioactivos (por ejemplo el U-238 no radioactivo puede captar una partícula y transformarse en plutonio radioactivo, $\mathrm{Pu}-239)$, o bien (2) divide elementos radioactivos, liberando más energía y partículas atómicas en una reacción en cadena. La reacción en cadena sostenida de un arma nuclear requiere altos porcentajes de U-235 o de plutonio. Sin embargo, para generar electricidad los reactores nucleares utilizan uranio natural, uranio enriquecido (menos de 5\% de U-235) o, en ocasiones, una mezcla de uranio y plutonio, llamada “MOX”. En una central nuclear la reacción en cadena libera calor para calentar agua y producir vapor, con el cual se genera electricidad.

El front end del ciclo del combustible nuclear incluye la extracción, la trituración y el enriquecimiento del uranio, y la fabricación de combustible (véase la Figura $\mathrm{N}^{\circ} 1$ ). A principios del siglo veinte el uranio era explotado como una fuente de radio (para la fabricación de pintura luminosa, como la que se usa en las esferas de los relojes, por ejemplo). El mineral de uranio se tritura y se lixivia con ácidos para producir un polvo de óxido de uranio, conocido como "pastel amarillo” (yellowcake). Para aumentar el porcentaje de U-235, el pastel amarillo es convertido en un gas (hexafluoruro de uranio, $\mathrm{UF}_{6}$ ) y enriquecido al separar el U-238 no radioactivo del U-235 radioactivo, de manera que el porcentaje de U-235 aumenta desde 0,7\% hasta entre $1 \%$ y $5 \%$. Luego del enriquecimiento, el gas de uranio es transformado en óxido de uranio $\left(\mathrm{UO}_{2}\right)$ y se procesa para formar barras de combustible, que se empaquetan e insertan en el reactor.

Cada 12 a 18 meses es necesario extraer algunas barras de combustible usado y reemplazarlas por barras de combustible nuevas, mientras que las barras restantes deben ser redistribuidas para equilibrar la energía radioactiva. El manejo de las barras de combustible extraídas constituye el back end del ciclo del combustible nuclear e incluye el manejo del combustible nuclear usado, el reprocesamiento opcional y la eliminación de los desechos radioactivos de alta actividad. Existen dos tipos de back ends. De un lado, cuando el ciclo de combustible es "abierto”, el combustible usado 
FIGURA N ${ }^{\circ}$ 1: $\quad$ CICLO DEL COMBUSTIBLE NUCLEAR

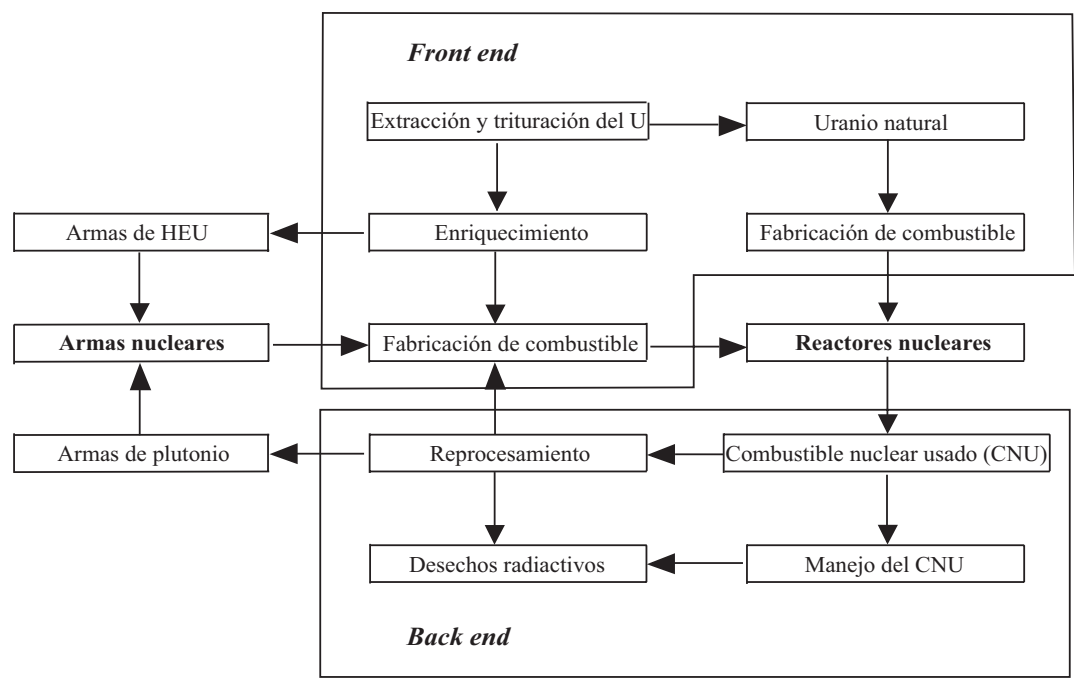

se enfría en la central atómica y luego se almacena o traslada a una instalación para su manejo centralizado. Allí es almacenado, o bien reembalado y enviado a un depósito geológico. Sólo algunos países están planificando la construcción de depósitos, por ejemplo los Estados Unidos en el monte Yucca. Del otro lado, el ciclo cerrado de combustible consiste en reprocesar el combustible usado para extraerle el uranio y el plutonio, los que son reciclados como combustible nuclear. Sólo unos pocos países reprocesan (o planean reprocesar) su combustible usado, por ejemplo Francia. La mayoría de los países están almacenando su combustible usado, esperando que decaiga. El análisis económico que sigue supone que el costo del back end abierto del ciclo del combustible nuclear es igual al valor presente de reprocesar a perpetuidad el combustible usado.

\subsection{Reactores nucleares comerciales}

Las centrales nucleares pueden clasificarse según cuatro características (véase la Figura $N^{\circ} 2$ ). En primer lugar, la reacción en cadena térmica de un reactor puede ser “rápida” o "lenta”. En un reactor rápido, la energía liberada durante la reacción en cadena no se atenúa, generándose más partículas nucleares que en un reactor térmico. Estas partículas pueden capturarse para convertir el uranio en plutonio, y por eso es que dichos reactores a veces reciben el nombre de "reactores de reproducción rápida” (fast breeder reac- 
FIGURA N ${ }^{\circ}$ 2: $\quad$ TIPOS DE REACTORES NUCLEARES

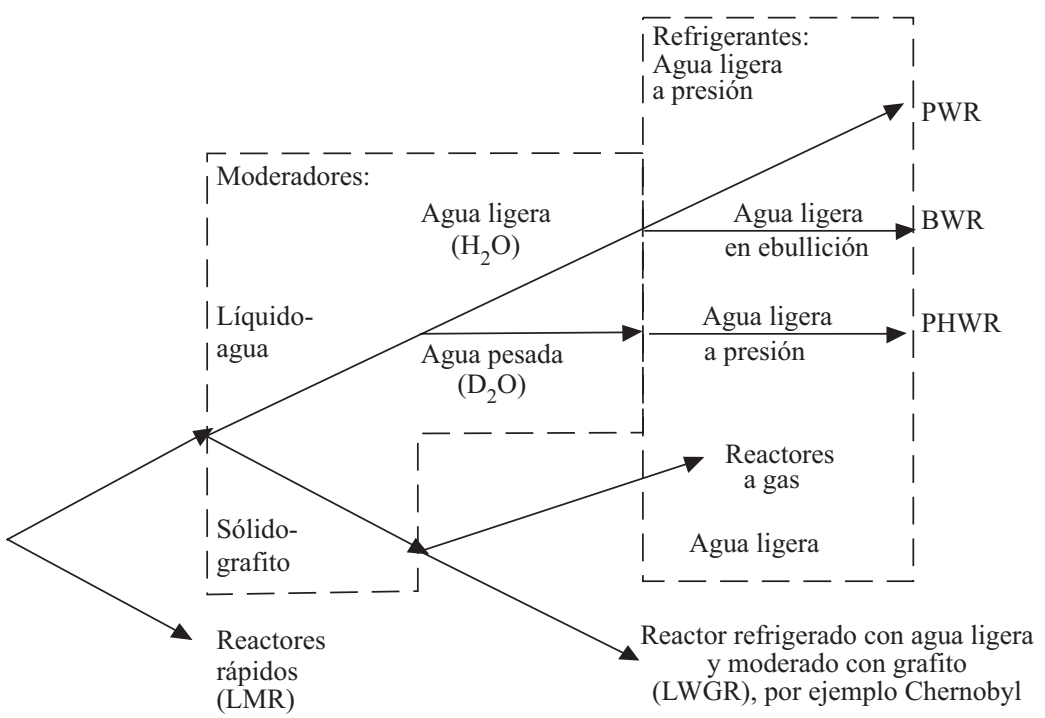

tors). También se conocen como "reactores de metal líquido” (LMR*), porque para enfriar el reactor se utilizan metales líquidos, por ejemplo, el sodio.

En segundo lugar, los reactores térmicos pueden distinguirse según el moderador de la reacción nuclear que absorbe las partículas nucleares; y, en tercer lugar, según el refrigerante escogido para eliminar el calor generado por la reacción en cadena. Los moderadores pueden ser agua ligera $\left(\mathrm{H}_{2} \mathrm{O}\right)$, agua pesada $\left(\mathrm{D}_{2} \mathrm{O}\right.$, donde la masa del deuterio, $\mathrm{D}$, es el doble de la masa del hidrógeno), o grafito (carbono). Los refrigerantes son agua (ligera o pesada) o gas (aire, dióxido de carbono o helio), los que permiten transferir el calor y obtener otras formas de energía, tales como la electricidad.

Por último, las centrales nucleares pueden distinguirse según utilicen un ciclo directo o indirecto para transferir la energía desde el reactor nuclear a la turbina que genera electricidad. El método directo permite que el refrigerante del reactor hierva, cambiando su estado desde líquido a gas (por ejemplo, de agua a vapor), impulsando directamente la turbina. El método indirecto transfiere la energía del reactor desde un circuito refrigerante primario a un circuito secundario a través de un generador a vapor, el que impulsa la turbina. El circuito primario suele ser presurizado, de modo que el agua refrigerante permanece en estado líquido.

* Las siglas de los reactores aparecen por su nombre en inglés. Véase Glosario de siglas al final del trabajo. (N. del E.) 
Aun cuando se han construido muchos reactores experimentales y militares, sólo hay cinco tipos de centrales nucleares para uso comercial (véase Organismo Internacional de Energía Atómica, OIEA, 2002):

1) Reactores de agua ligera (LWR), incluidos (a) los reactores refrigerados y atenuados con agua ligera a presión (PWR) producidos en Estados Unidos, Europa y Japón, y los reactores atenuados y refrigerados con agua a presión del tipo WWER, desarrollados en la ex Unión Soviética y la actual Rusia, y (b) reactores atenuados y refrigerados con agua ligera en ebullición (BWR), fabricados principalmente por General Electric en los Estados Unidos.

2) Reactores de agua pesada (HWR), incluido el reactor atenuado y refrigerado con agua pesada a presión (PHWR), producido principalmente en Canadá (CANDU).

3) Reactores refrigerados con gas y atenuados con grafito (GCR), incluidos los reactores de alta temperatura refrigerados con gas y atenuados con grafito (HTGR), como el pequeño reactor modular (small pebblebed modular reactor, PBMR) que está siendo desarrollado por Sudáfrica.

4) Reactores refrigerados con agua ligera y atenuados con grafito (LWGR), incluido el reactor RBMK de diseño soviético, por ejemplo el de Chernobyl.

5) Reactores de metal líquido (LMR).

Muchos programas nucleares han previsto el despliegue secuencial de tres tecnologías nucleares: (tecnología 1) reactores de agua pesada refrigerados con gas, o de agua ligera atenuados con grafito, los cuales usan uranio natural; (tecnología 2) reactores de agua ligera y de alta temperatura refrigerados con gas, los cuales usan uranio enriquecido o $\mathrm{MOX}$, y (tecnología 3) reactores de metal líquido que utilizan uranio enriquecido, MOX o plutonio. Hasta comienzos de los años setenta, los reactores refrigerados con gas tenían la participación de mercado más alta. Sin embargo, a partir de entonces los reactores de agua ligera comenzaron a dominar la industria internacional de la energía nuclear, y continúan dominándola hoy.

En el Cuadro $N^{0} 1$ se muestra que en cada región, excepto Latinoamérica, existe una tecnología predominante: (1) reactores de agua ligera en Estados Unidos, la ex Unión Soviética y Europa oriental, Francia, Europa occidental y Asia oriental; (2) reactores refrigerados con gas en Gran Bretaña, y (3) reactores de agua pesada en la Commonwealth británica. En todas las regiones se han construido reactores de agua pesada; también se han construido reactores de metal líquido en todo el mundo, excepto en América Latina. 


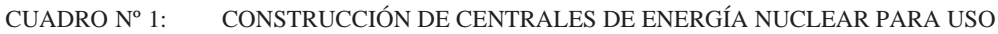
COMERCIAL POR REGIÓN (AL AÑO 2002)

\begin{tabular}{lrrrrrrrr}
\hline & $\begin{array}{c}\text { Estados } \\
\text { Unidos }\end{array}$ & $\begin{array}{c}\text { Gran Bretaña } \\
\text { y la } \\
\text { Commonwealth }\end{array}$ & Francia & $\begin{array}{c}\text { Europa } \\
\text { occidental }\end{array}$ & EUS/EO/AC & $\begin{array}{c}\text { Asia } \\
\text { oriental }\end{array}$ & $\begin{array}{c}\text { América } \\
\text { Latina }\end{array}$ & Total \\
\hline PWR & 79 & 4 & 59 & 39 & 1 & 52 & 3 & 237 \\
BWR+ & 43 & 2 & 0 & 27 & 2 & 38 & 2 & 114 \\
WWER & 0 & 0 & 0 & 0 & 66 & 0 & 0 & 66 \\
HWR & 1 & 39 & 1 & 3 & 3 & 7 & 3 & 57 \\
GCR & 2 & 41 & 8 & 4 & 0 & 1 & 0 & 56 \\
LWGR & 0 & 0 & 0 & 0 & 24 & 0 & 0 & 24 \\
RML & 1 & 2 & 2 & 1 & 2 & 1 & 0 & 9 \\
Total & 126 & 88 & 70 & 74 & 98 & 99 & 8 & 563 \\
\hline
\end{tabular}

Nota: EUS/EO/AC: Ex Unión Soviética / Europa oriental / Asia central.

Fuente: OEIA (2002).

\subsection{La energía nuclear en América Latina}

Tres países de América Latina, México, Argentina y Brasil, han desarrollado programas comerciales de energía nuclear en pequeña escala. Entre 1976 y 1995 México construyó dos reactores General Electric del tipo BWR de 700 MW de potencia.

Argentina, con ayuda estadounidense, construyó su primer reactor de investigación en 1958, y tres más durante los años sesenta. En 1968 los alemanes iniciaron la construcción de un reactor de agua pesada de 350 MW, el que comenzó a operar comercialmente en 1974. Durante los años setenta se construyó una planta de enriquecimiento en Pilcaniyeu, y una instalación de reprocesamiento en Ezeiza. En 1974 se iniciaron las obras de una segunda central nuclear (una CANDU de $600 \mathrm{MW}$ ), las que terminaron en 1984.

Brasil comenzó a explotar uranio en los años cuarenta e intentó desarrollar el ciclo completo del combustible nuclear entre 1950 y 1990. A fines de los años cincuenta y durante los años sesenta se construyeron reactores de investigación con ayuda estadounidense. En 1971 se empezó a construir un PWR Westinghouse de 650 MW (Angra 1), y las obras concluyeron en 1984. Para adquirir la tecnología para producir combustible nuclear, Brasil negoció con Alemania la compra de varias centrales nucleares, una instalación para enriquecer uranio y una planta piloto de reprocesamiento. La construcción de Angra 2 empezó en 1976 y prosiguió por 24 años hasta que entró en funcionamiento comercial en 2000. Si bien ninguna de las restantes 
instalaciones alemanas se completó, Brasil comenzó a enriquecer uranio en Resende, y dentro de pocos años Angra 1 y Angra 2 serán abastecidas con combustible local (véase Cabrera-Palmer y Rothwell, 2008).

\section{Antes de construir la primera central es necesario desarrollar la estructura regulatoria nuclear}

Si bien podría estar gestándose un renacimiento nuclear en aquellos países donde actualmente operan centrales atómicas, ninguno tiene experiencia suficiente para construir los reactores de agua ligera de la siguiente generación. A medida que dicho renacimiento se desarrolle, el precio del combustible para reactores de agua ligera podría fluctuar. Sin embargo, los determinantes de los costos de operación y los costos del combustible para reactores avanzados de agua ligera (ALWR) son bien conocidos porque la tecnología es muy similar a la actual.

Sin embargo, se desconocen los costos de construcción de las nuevas centrales nucleares (aunque se conocen los de aquellas centrales que actualmente se están construyendo, porque ya se han licitado los contratos). En el pasado reciente el precio de las centrales nucleares ha aumentado (al igual que los de todas las centrales eléctricas) debido al aumento del precio internacional de los materiales y las maquinarias de construcción. Si a eso se suma el clima de incertidumbre que reina en los mercados de capital, el costo total de las inversiones de capital en energía nuclear resulta impredecible, particularmente cuando se trata de la primera central construida en un país en vías de desarrollo.

Además, si bien algunos países han construido y operado reactores de investigación, no necesariamente cuentan con la infraestructura necesaria para regular a una industria de energía nuclear. Dicha infraestructura se describe en una publicación del Organismo Internacional de Energía Atómica (OIEA, 2007). El informe de la OIEA (2007) es extraordinariamente detallado y debería leerse con cuidado. (En el Apéndice se reproduce el "Índice de materias” de esa publicación). De todos modos, el proceso básico que conduce a construir y operar una central nuclear se divide en tres etapas:

Primera Etapa: al completarla, el país se encuentra dispuesto a comprometerse, con conocimiento de causa, con un programa nuclear".

Segunda Etapa: al completarla, el país se encuentra en condiciones de llamar a licitación para construir la primera central nuclear.

Tercera Etapa: al completarla, el país se encuentra en condiciones de poner en servicio y operar la primera central nuclear. 
Para resumir: antes de construir una central nuclear, Chile debe emitir un permiso de obras. Organismos tales como la Comisión Reguladora de la Energía Nuclear (Nuclear Regulatory Commission, NRC) de los Estados Unidos podrían ayudar a crear un organismo regulador nacional. Al mismo tiempo, el nuevo regulador chileno podría emular los preocedimientos de la NRC para autorizar la construcción de un reactor. Sin embargo, es indispensable que el nuevo regulador chileno sea capaz de adaptar las normas de seguridad a las condiciones particulares de Chile —a su topografía, sismología y ecosistemas-. Además, para manejar el combustible nuclear se necesita crear y capacitar a un organismo civil o militar que actúe de manera coordinada con la OIEA.

Para crear dichos organismos y adquirir la competencia técnica necesaria, un país debe invertir grandes montos antes de autorizar la construcción de la primera central nuclear. Estas inversiones, sin embargo, son hundidas. Polonia, por ejemplo, desarrolló esta infraestructura durante los años ochenta, después de negociar con la Unión Soviética la construcción de una central nuclear. Pero el proyecto fue cancelado tras la disolución de la Unión Soviética, y si bien partes de dicha infraestructura (por ejemplo, los conocimientos profesionales especializados) aún se encuentran disponibles, la mayoría de las inversiones realizadas durante la era soviética se perdieron.

El Cuadro $\mathrm{N}^{\circ} 2$ identifica las tareas básicas para regular y luego construir, en cuatro períodos de creciente complejidad y costo, la primera central nuclear. El plazo de los primeros tres períodos es alrededor de doce años (por ejemplo, desde el 2009 hasta 2020). La duración de cada una de las tres primeras etapas puede acortarse o extenderse en función del presupuesto y del grado de compromiso. Durante el cuarto período se construye la central nuclear, lo cual podría tomar entre 4 y 24 años, tal como ocurrió con Angra 2 en Brasil. Los primeros dos períodos descritos en el Cuadro $\mathrm{N}^{\circ}$ 2 corresponden a la Primera Etapa del informe OIEA (2007). La Segunda Etapa del informe OIEA corresponde al tercer período, y la Tercera Etapa, al cuarto período. Pese a que la evaluación del costo de cada tarea va más allá del ámbito de este trabajo, en la sección 5 presento algunas estimaciones.

Un país se comprometerá con este proceso dependiendo de: 1) la intensidad del compromiso “con conocimiento de causa” de la opinión pública con la energía nuclear; y 2) los costos y beneficios de construir la primera central nuclear. El beneficio principal es la generación de electrici- 
CUADRO N 2: TAREAS ESTRATÉGICAS PARA LA PRIMERA CENTRAL NUCLEAR DE UN PAÍS

Primer período: 4 años de estudio y debate que llevan a completar la Etapa 1 de la OIEA

Definir la regulación que se exigirá para autorizar una central nuclear.

Evaluar los aspectos económicos de las etapas restantes.

Definir los criterios para seleccionar la tecnología y el combustible.

Definir los criterios para seleccionar el lugar donde se construirá una central nuclear.

Revisar las políticas de control de desechos nucleares y de emisiones de carbono.

Evaluar con sondeos de opinión pública si existe consenso en que la opción nuclear es conveniente.

\section{Segundo período: 4 años de creación de instituciones}

Crear las instituciones reguladoras e iniciar la capacitación del personal.

Evaluar los aspectos económicos de las etapas restantes.

Elegir la tecnología y el combustible.

Elegir al arquitecto, al ingeniero y al jefe de obras.

Elegir y definir los emplazamientos potenciales.

Regular el manejo de los desechos nucleares de baja radioactividad.

Tercer período: 4 años de preparación de la construcción, que llevan a completar la Etapa 2 de la OIEA

Evaluación económica del proyecto y de las fuentes de financiamiento.

Desarrollar proveedores locales de insumos.

Autorizar el emplazamiento de la central a los proveedores locales y la tecnología.

Negociar los contratos eléctricos.

Planificar el financiamiento y el refinanciamiento de la construcción de la obra.

Obtener las servidumbres para tender líneas de alta tensión.

Regular el manejo de los desechos nucleares de alta radioactividad.

Llamar a licitación y evaluar las propuestas.

Cuarto período: 4 años para construir la central, los cuales llevan a completar la Etapa 3 de la OIEA.

Supervisar la construcción, la tecnología y a los proveedores de equipos.

Actualizar la evaluación económica y el financiamiento de la construcción. Implementar el financiamiento y refinanciar la construcción.

Construir instalaciones para almacenar desechos nucleares radioactivos.

Autorizar el funcionamiento de la central ya terminada. 
dad limpia, aunque el desarrollo industrial inducido por la energía atómica podría producir beneficios indirectos. En la siguiente sección se analizan los costos primarios de la energía nuclear ${ }^{1}$.

\section{El costo monómico de generar electricidad ${ }^{2}$}

El principal obstáculo que se debe superar para construir la primera central nuclear es su costo, que es muy grande cuando se compara con alternativas más baratas (pero más contaminantes) basadas en combustibles fósiles. Hoy en día, cuando los países eligen entre centrales alimentadas con combustibles fósiles, prefieren el carbón por su amplia disponibilidad y la estabilidad de su precio. Esto contrasta con el gas natural, cuyo precio es elevado y sumamente volátil. Sin embargo, el costo del carbón bajo un régimen internacional de control de los gases de efecto invernadero es incierto. En la actualidad no se conoce con precisión el costo social del daño causado por las emisiones de dióxido de carbono $\left(\mathrm{CO}_{2}\right)$, o cuál podría ser el precio de mercado de un permiso para emitirlo. En este trabajo me referiré a cualquier pago asociado a la emisión de $\mathrm{CO}_{2}$ como " $\mathrm{CO}_{2} \mathrm{ST}$ ", es decir costo del dióxido de carbono (aun cuando el mecanismo de control de emisiones de $\mathrm{CO}_{2}$ podría involucrar la aplicación de un impuesto a las emisiones o de un sistema de permisos transables y topes máximos de emisión). Una central a carbón tipo produce normalmente alrededor de siete millones

${ }^{1}$ Por cierto, un análisis más completo incluiría la seguridad de las centrales, las consecuencias ambientales de la energía nuclear, como también aspectos relacionados con la no proliferación. El historial de seguridad de los reactores de agua ligera es excelente, pero para lograr tales estándares es necesario adoptar una "cultura de la seguridad”; es decir, procedimientos de operación tales que la seguridad siempre es prioritaria. En este trabajo se presume que Chile será capaz de desarrollar una cultura de seguridad nuclear. El combustible radioactivo usado debe ser monitoreado de por vida. El costo de este proceso debe compararse con el costo de capturar los contaminantes emitidos por centrales que queman combustibles fósiles. Actualmente la energía nuclear internaliza el costo de sus desechos, mientras que la generación con combustibles fósiles no internaliza el costo del daño causado por el dióxido de carbono. Por último, es improbable que Chile enfrente problemas por proliferación nuclear si se abstiene de comprar combustible nuclear a países fabricantes de armas nucleares (tales como Francia, Rusia o Estados Unidos).

${ }^{2}$ El siguiente análisis se efectúa en dólares estadounidenses de 2005, principalmente porque, en su Panorama Anual de la Energía, la EIA de Estados Unidos (US Energy Information Administration) ha estado utilizando dólares de 2005 en sus últimos análisis; véase, por ejemplo, la Tabla 39 en EIA (2007). Además, debido a la volatilidad observada desde entonces, no existe un índice de precios confiable para actualizar los precios al año 2008 y siguientes. Por consiguiente, mi cálculo es ilustrativo sobre cómo habría que realizar un análisis más completo y actualizado aunque no definitivo. Por último, las conclusiones cualitativas no se ven afectadas en modo alguno por el hecho de escoger los dólares estadounidenses de 2005 como base cuantitativa. 
de toneladas de $\mathrm{CO}_{2}$ al año. La Figura $\mathrm{N}^{\circ} 3$ muestra el precio de un permiso de emisión de $\mathrm{CO}_{2}$ en la Unión Europea durante 2005. (El 1 de octubre de 2008, el precio de un permiso de emisión ascendía a $\bullet 23,10 / \mathrm{CO}_{2}$ o aproximadamente US\$ 32,32/t $\mathrm{CO}_{2}$ ). Por tanto, si el $\mathrm{CO}_{2} \mathrm{ST}$ es de US\$ 25 (en dólares de 2005) por tonelada de $\mathrm{CO}_{2}$, los propietarios de la central a carbón podrían tener que pagar US\$ 175 millones al año por emitir o, alternativamente, capturar las emisiones. Si bien ya se tiene experiencia para calcular las distribuciones estadísticas de los precios de los permisos de emisión, en el futuro (por ejemplo en 2020) tendremos una idea aún más precisa.

\section{FIGURA N ${ }^{\circ}$ 3: $\quad$ ÍNDICE EUROPEO DE $\mathrm{CO}_{2}$, EURO/TONELADA DE $\mathrm{CO}_{2}, 2005$}

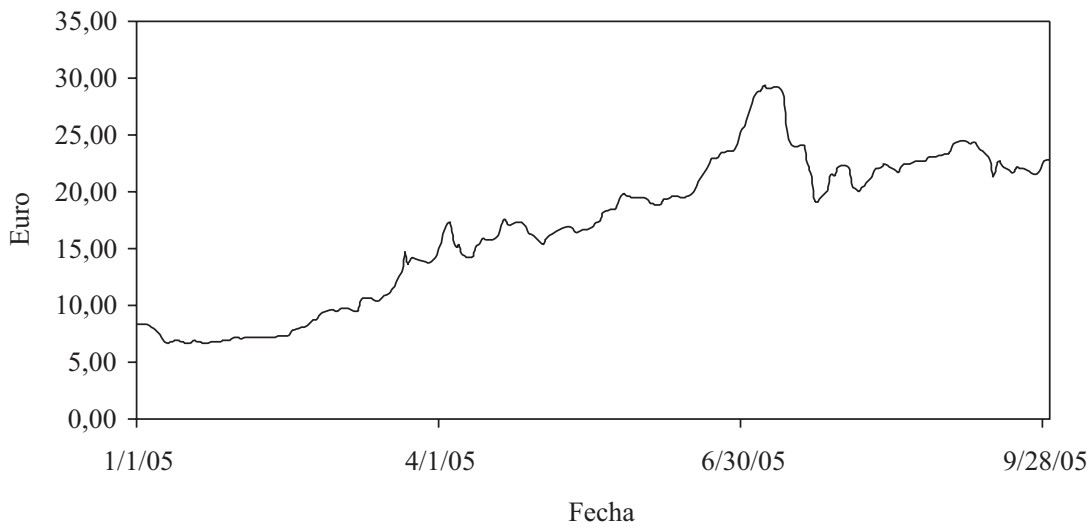

Fuente: http://www.eex.com/en/Download/Market\%20Data.

En vista de esta incertidumbre, varias economías en vías de desarrollo, con capacidad instalada de generación superior a los 10.000 MW, están considerando la energía nuclear.

Sin embargo, para que una red de transmisión sea confiable es preciso que la central más grande no represente más del $10 \%$ de la capacidad (Vergara, 2007, p. 77). Por eso, mientras no existan centrales nucleares pequeñas (lo que podría ocurrir recién hacia 2020; por ejemplo, el pequeño reactor nuclear sudafricano refrigerado con gas), la energía nuclear no es práctica para sistemas de menos de 10.000 MW*.

El costo de construir y operar plantas generadoras de electricidad es ampliamente discutido (véase, por ejemplo, NEA 2005). Con todo, el costo real previsto de construir una planta generadora de electricidad dada siem-

* Hoy día la capacidad instalada en el Sistema Interconectado Central (SIC) de Chile es poco más de 8.000 MW. (N. del E.) 
pre se mantiene confidencial (ya que el hecho de conocer este dato supone una ventaja competitiva), mientras que la mayoría de las fuentes de información pública sobre costos quedan obsoletas rápidamente o son incompletas. Además, si bien al menos una decena de estudios proyectan los costos de la generación con combustibles fósiles o con energía nuclear, pocas veces se ha modelado la magnitud de la incertidumbre de esas estimaciones de costos. En esta sección se examinan las estimaciones de costos sobre la base de EIA (2007). En la sección 4 se analiza el método para modelar la incertidumbre con que se estima el valor presente neto del costo de las centrales nucleares y las centrales a carbón, y se evalúa la alternativa de construir una central nuclear. Esta información puede servir de guía para determinar si vale la pena invertir en la institucionalidad regulatoria necesaria para tener la opción de construir una central nuclear en algunos años más.

El costo monómico por megawatt-hora (MWh) es la principal medida usada en la industria eléctrica para comparar los costos de distintas tecnologías de generación. Es igual al valor presente del costo total de capacidad y operación y de combustible durante toda la vida de la planta dividido por el valor presente del total de energía generada ${ }^{3}$. Para calcular una serie comparable de costos monómicos para varias tecnologías de generación de electricidad, EIA (2007) entrega un conjunto coherente de supuestos (véase el "Módulo del Mercado de la Electricidad" de su "Sistema Nacional de Modelación Energética”, en Panorama Anual de la Energía. Estos valores son similares a los reportados en el capítulo 3, "Costos de generación en centrales a carbón, a gas y nucleares”, NEA 2005).

Si bien el Cuadro No 39 de EIA (2007) señala la potencia en MW, el tiempo necesario para construir una central, los costos de construcción, los factores asociados a imprevistos, los costos totales de construcción, los costos variables de operación y mantenimiento por kilowatt-hora, los costos fijos de operación y mantenimiento por kilowatt, como también la tasa de consumo de calor para 19 tecnologías de generación, no se especifican los costos de capital o de combustible.

\footnotetext{
${ }^{3}$ Para definir el costo monómico, supongamos que el costo total anual, $C T$, es igual a $p_{K} K+(O \& M)_{t}+p_{F} F_{t}+W_{t}$, donde $K$ es el costo total de la inversión de capital (CTIC, definido en EMWG, 2007) medido en millones de dólares de 2005, y $p_{k}$ es la tasa anual de costo de capital; $O \& M_{t}$ es costo anual de operación y mantenimiento; $F_{t}$ es el combustible consumido anualmente, $p_{F}$ es el precio del combustible; y $W_{t}$ es el costo anual del manejo de los desechos nucleares. El costo monómico es igual a [ $\Sigma C T_{t}$ $\left.(1+r)^{-t}\right] /\left[\Sigma M W h_{t}(1+r)^{-t}\right]$, donde la suma total supera la vida comercial de la instalación (la totalidad de los costos de construcción se actualiza a la fecha de operación comercial). $M W h_{t}$ es la producción anual, y $r$ es la tasa de descuento apropiada.
} 
Por consiguiente, los parámetros a especificar son (1) el costo de capital durante la construcción y la operación; (2) el costo del combustible, y (3) el costo del manejo de desechos. (Se supone que todas las centrales tienen una vida económica de cuarenta años, y un factor de planta promedio de $90 \%$ ). En el Cuadro $\mathrm{N}^{\circ} 3$, columna 3, se presenta el costo monómico de la "tecnología nuclear avanzada" estimado por la EIA. La columna 4, presenta el costo monómico de una central a carbón y la columna 5 presenta el costo monómico del "ciclo combinado avanzado gas/petróleo" (CCGT). (Aun cuando estos valores ya están obsoletos, los valores relativos seguramente siguen siendo correctos; por ejemplo la energía nuclear es la tecnología más cara si no se cobra por emitir $\mathrm{Co}_{2}$, pero la más barata cuando se cobra. Es preciso actualizar constantemente esas estimaciones.)

En estos cuadros se supone que el costo de capital es 7\% real y 10\% nominal. Sin embargo, debido a la incertidumbre a la que está sujeta la construcción de nuevas centrales nucleares, se añade una prima por riesgo de 5\% real durante la construcción (Rothwell, 2006), lo cual arroja un costo del capital de $12 \%$ real, o $15 \%$ nominal. Siguiendo a Lapuerta y Brown (2007), se supone que, una vez terminadas las obras de la central, los gastos de construcción, incluido el interés durante la construcción (IDC), son refinanciados a una tasa de $7 \%$ real.

Con respecto a los precios de los combustibles, se supone lo siguiente: (1) el precio monómico del combustible nuclear es US\$ 7,67/MWh más US\$3/MWh por manejo de desechos radioactivos y por la demolición de la central cuando cumple su vida útil (véase Rothwell, 2006); (2) el precio previsto del carbón es de US\$2/M BTU (millones de BTU en dólares de 2005, equivalentes a $\$ 1,90 / G J$; véase la Tabla 3.3 en NEA, 2005), donde se supone que el rango de precios del carbón en 2020 (en dólares de 2003) va desde US\$ 1,16/GJ en Corea del Sur hasta US\$ 2,27/GJ en Finlandia, y (3) el precio previsto del gas natural es de US\$ 5/M BTU (en dólares de 2005), lo cual equivale a \$ 4,74/GJ (véase la Tabla 3.6 en NEA, 2005), donde el rango de precios del gas natural para el año 2020 (en dólares de 2003) va desde US\$ 4,18/GJ en Francia hasta US\$ 6,42/GJ en Italia. Por último, el costo del $\mathrm{CO}_{2}$ en 2020 se estima en US\$ 25 por tonelada en dólares de 2005.

Con respecto al costo de emitir $\mathrm{CO}_{2}$, consideremos la siguiente declaración del European Climate Exchange (http://www.europeanclimateexchange. com/default_flash.asp):

El enfoque de límites de emisión y permisos (cap and trade) que está siendo utilizado en el Régimen de Comercio de Derechos de Emisión de la Unión Europea [European Union Emission Trading Scheme, EU ETS] fija un tope o cantidad 
CUADRO N N $^{\circ}$ COMPARACIÓN ENTRE COSTOS DE UN REACTOR ALWR, UNA PLANTA A CARBÓN Y UN CICLO COMBINADO O GAS NATURAL CCGT, BASADA EN EIA (2007, TABLA 39)

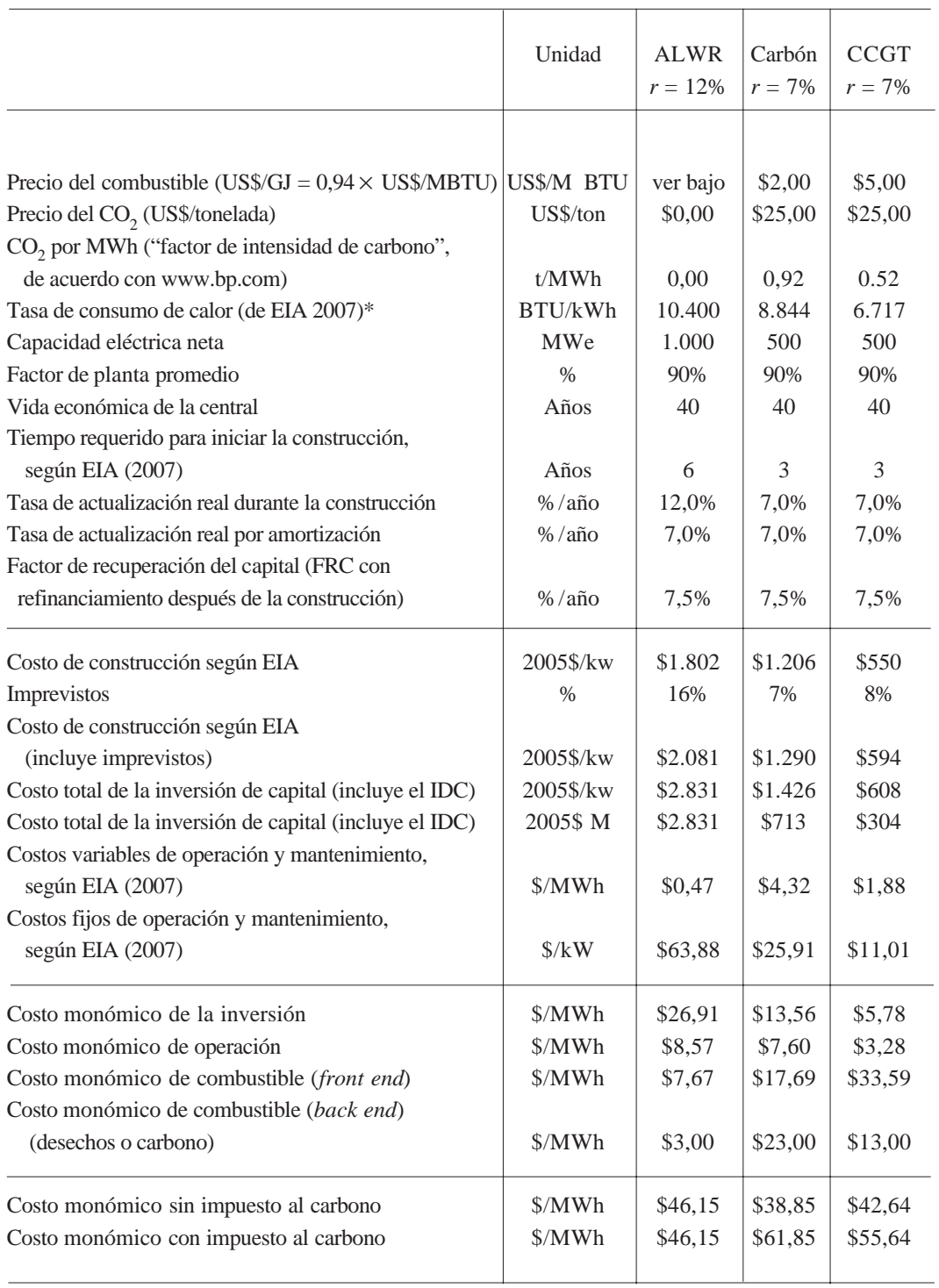

* Los tamaños típicos de las plantas han sido modificados para facilitar la comparación. La EIA supone tamaños de 1.350 MW para centrales nucleares, 600 MW para centrales a carbón y 400 MW para centrales a gas natural. 
máxima global de emisiones por período de cumplimiento. Las empresas reciben cuotas de emisión que representan su meta o "tope" durante un período. Al final de ese período deben entregar una cantidad de permisos suficiente que coincida con el total de sus emisiones durante el período. Si esta cifra es inferior al tope, disponen de permisos para vender; si no, deben comprar permisos a empresas que han excedido sus límites de reducción de emisiones. Cada permiso de emisión le permite al titular emitir una tonelada de $\mathrm{CO}_{2}$. Si a la fecha de cumplimiento un operador no tiene suficientes cuotas para cubrir su cifra total de emisiones, se le aplicará una multa de - 40 por tonelada excedida para la Primera Fase 2005-2007, la que se elevará a • 100 en la Segunda Fase 2008-2012.

Teniendo en cuenta estas multas y el valor del dólar, US\$ 25 por tonelada de $\mathrm{CO}_{2}$ parece una cifra razonable para el 2020, pero no es posible conocer qué países participarían en un acuerdo internacional como éste.

El Cuadro $N^{\circ} 3$ muestra que el costo económico de la energía nuclear es 19\% mayor que el del carbón y 8\% mayor que el del gas natural si no se cobra por emitir $\mathrm{CO}_{2}$. Sin embargo, el costo de la energía nuclear es 25\% menor que el del carbón y 17\% que el del gas natural, si se incluye el costo de US\$ 25 por tonelada emitida de $\mathrm{CO}_{2}$. (Por cierto que estos costos no incluyen el de la institucionalidad regulatoria de la energía nuclear.) Aun cuando estos resultados dependen de los supuestos, este ejemplo demuestra que la energía nuclear podría ser económicamente competitiva en un mundo en que se imponen restricciones a las emisiones de carbono.

\section{El valor actual neto de una nueva central nuclear}

Aunque el análisis del costo monómico es ilustrativo, antes de que una firma comience a construir una central nuclear debe demostrarle a sus accionistas que el valor presente neto (VAN) del proyecto es positivo ${ }^{4}$. Para ello hay que proyectar los flujos de fondos positivos y negativos, y actualizarlos aplicando la tasa de descuento que corresponda. En esta sección se discute la forma en que se debe calcular el VAN de una central nuclear y representar su distribución de probabilidades.

Los gastos de construcción se actualizan hasta el inicio de la operación (por ejemplo el año 2026) descontándolos al 12\% real (incluida en la inversión-total-de-capital como “interés durante la construcción”), y los

${ }^{4}$ El VAN equivale a $\left[\Sigma\left(p_{Q} Q_{t}-O \& M_{t}-p_{F} F_{t}-W_{t}\right) /(1+r)^{-t}\right]-K$, donde $p_{Q}$ es el precio de la electricidad, y las demás variables son las mismas que en la nota 3. 
ingresos de explotación se actualizan hasta el inicio de la operación utilizando una tasa del 7\% real. Los ingresos de explotación corresponden a la cantidad de MWh generados multiplicada por el precio por MWh. Como punto de referencia, el precio de la electricidad se supone que es igual al costo monómico de generar electricidad con una central a carbón, porque todos los países, incluido Chile, tienen acceso a los mercados de carbón y su tecnología. El VAN se calcula a 2026 (el inicio de la operación), a 2020 (el inicio de la construcción) y a 2008 (el inicio de los preparativos).

Adicionalmente, los costos estimados en 2005 probablemente ya están obsoletos, en particular cuando se proyectan al 2020. Chupka y Basheda (2007) muestran que entre 2003 y 2006 el costo de construir plantas de electricidad aumentó en 75\%. Para explicar estas tendencias y por qué la EIA las ignora, en EIA (2007, p. 36) se señala lo siguiente:

Durante los últimos años, los costos de construcción han sido volátiles. Parte de la volatilidad puede deberse al alza de los precios de la energía. Los precios del hierro y del acero, del cemento y del concreto - materias primas utilizadas intensivamente en la construcción de centrales - subieron abruptamente entre 2004 y 2006, y se ha informado de una situación de escasez. No se sabe con certeza de qué manera pueden influir esas fluctuaciones de precio en el costo o en el ritmo de los nuevos proyectos emprendidos en el ámbito energético, y en las proyecciones a 25 años en AEO (2007) se ignoran las variaciones de corto plazo de los precios de las materias primas. En las industrias energéticas la mayoría de los proyectos deben planificarse con mucha anticipación y requieren un largo período previo al inicio de la construcción, lo cual puede aminorar el impacto de las tendencias de corto plazo.

También el precio de los permisos de emisión de $\mathrm{CO}_{2}$ podría ser incluso más volátil. Roques y otros (2006, p. 13) suponen que la volatilidad anual es 30\% (Figura $\mathrm{N}^{\circ}$ 3). Véase, por ejemplo, la volatilidad de los datos históricos en el European Carbon Exchange (Bolsa Europea de Intercambio de Bonos de Carbono). Como señala Nordhaus (2001, p. 30):

Una de las posibles preocupaciones respecto de la actual estructura del Protocolo de Kioto es que inducirá gran volatilidad de los precios de los permisos. La volatilidad puede apreciarse en el historial de los precios de los permisos de emisión de $\mathrm{SO}_{2}$, que han sido mucho más volátiles que el IPC o, incluso, que los precios bursátiles. 
Ello se debe a que tan pronto como se impone una cantidad límite, las transacciones internacionales pueden dar lugar a una competencia encarnizada en vista de las multas impuestas a quien excede el tope.

En vista de todo lo anterior, para analizar la varianza del VAN de una central nuclear, supongamos lo siguiente:

1) Entre el 2005 y el 2020 el costo de construcción de centrales nucleares aumenta en $4 \%$ real anual con una desviación estándar de $2 \%$; el costo de construcción de centrales a carbón aumenta a una tasa real de $2 \%$ anual con una desviación estándar de $1 \%$; ello se traduce en un costo monómico de aproximadamente US\$ 42/MW para centrales nucleares, y de US\$17/MW para centrales a carbón el 2020, en dólares de 2005 (estas cifras están más cercanas a los costos anunciados en 2008; véase Ray, 2008, donde se cita a Progress Energy y a Florida Power \& Light).

2) El precio del combustible nuclear y el precio del carbón aumentan en 1,5\% (real) entre el 2020 y el 2065; con ello se duplica el costo del combustible durante el período de vida útil de cuarenta años.

3) Los precios del combustible nuclear y del carbón siguen una distribución probabilística con una media de 0 y una desviación estándar de US\$ 0,17, calculada con información de precios internacionales del carbón utilizando una estimación del error cuadrático medio dentro de un proceso autorregresivo de primer orden (véase Rothwell, 2006, p. 45).

4) $\mathrm{CO}_{2} \mathrm{ST}$ sigue una distribución uniforme entre US $\$ 0$ y US $\$ 50$ por tonelada el 2020, y entre el 2020 y el 2065 sigue un proceso autorregresivo con desviación estándar igual a 17\%, produciendo una volatilidad de 30\%, tal como se supone en Roques y otros (2006).

En el Cuadro $\mathrm{N}^{\circ} 4$ se presenta un análisis determinista del flujo de fondos. El costo total de la inversión de capital es de US\$4.645/kWe, o US\$ 4.645 millones para una central de 1.000 MW (incluido el interés durante la construcción, IDC). El VAN (en dólares de 2005 en 2008) es US\$ 163 millones excluido el costo por emisión de $\mathrm{CO}_{2}$, y es una cifra positiva de US\$ 602 millones si se incluye dicho costo. Además, las Figuras $\mathrm{N}^{\mathrm{os}} 4$ y 5 presentan los resultados de 100.000 simulaciones (en las que se utilizó el programa Cristal Ball ${ }^{\circledR}$ ). Ello ofrece una representación de la distribución probabilística para el VAN, incluidos y excluidos el costo de las emisiones de carbono.

La Figura $N^{\circ} 4$ muestra que la probabilidad de que una central nuclear arroje un VAN positivo cuando se ignoran los costos por emisión de $\mathrm{CO}_{2}$ es 0,31 (31\%); la esperanza del valor presente es menos US\$ 131 millones. Sin embargo, esta probabilidad aumenta a 0,8 (80\%) cuando se incluyen los costos de emitir $\mathrm{CO}_{2}$, en tanto que el valor presente esperado es US\$ 603 millones (véase la Figura $N^{\circ}$ 5). Es más, debido a la volatilidad de los 
CUADRO N N$^{\circ}$ 4: VALOR ACTUAL NETO DE UNA CENTRAL DE ENERGÍA NUCLEAR, COMPARADO CON EL DE UNA CENTRAL A CARBÓN

Análisis del flujo de fondos (millones de dólares de 2005)

Precio de la electricidad $=$ costo del carbón

Valor actual neto (VAN el 2026)

Valor actual neto (VAN el 2020)

Valor actual neto (VAN el 2008)

Costo total de la inversión de capital

\begin{tabular}{|c|c|c|c|}
\hline Actividad & Año & $\begin{array}{c}\text { Flujo de fondos } \\
\text { actualizado }\end{array}$ & $\begin{array}{c}\text { Flujo de fondos } \\
\text { actualizado }\end{array}$ \\
\hline Inicio de la construcción & 2020 & $-\$ 1.009$ & $-\$ 1.009$ \\
\hline Año de construcción & 2021 & $-\$ 901$ & $-\$ 901$ \\
\hline Año de construcción & 2022 & $-\$ 804$ & $-\$ 804$ \\
\hline Año de construcción & 2023 & $-\$ 718$ & $-\$ 718$ \\
\hline Año de construcción & 2024 & $-\$ 641$ & $-\$ 641$ \\
\hline Año de construcción & 2025 & $-\$ 572$ & $-\$ 572$ \\
\hline Año de operación & 2026 & \$ 272 & $\$ 447$ \\
\hline Año de operación & 2027 & \$ 255 & $\$ 419$ \\
\hline Año de operación & 2028 & \$ 239 & \$ 393 \\
\hline Año de operación & 2029 & $\$ 225$ & $\$ 368$ \\
\hline Año de operación & 2030 & $\$ 211$ & $\$ 345$ \\
\hline Años de operación 2031-2060 & $\ldots$ & $\ldots \ldots$ & $\ldots .$. \\
\hline Año de operación & 2061 & $\$ 31$ & $\$ 47$ \\
\hline Año de operación & 2062 & $\$ 29$ & $\$ 44$ \\
\hline Año de operación & 2063 & $\$ 27$ & $\$ 42$ \\
\hline Año de operación & 2064 & $\$ 26$ & $\$ 39$ \\
\hline Año de operación & 2065 & $\$ 24$ & $\$ 37$ \\
\hline
\end{tabular}

$\begin{array}{ll}\text { Sin costo } & \text { Con costo } \\ \text { por } \mathrm{CO}_{2} & \text { por } \mathrm{CO}_{2}\end{array}$

$\$ 38,85 \$ 61,85$

$-\$ 534 \$ 1.968$

-\$356 \$1.311

-\$163\$ \$602

$\$ 4.645 \$ 4.645$

precios y a su naturaleza autorregresiva (en virtud de la cual el precio en un determinado año depende del precio del año anterior), es posible que la inversión en una central nuclear obtenga un retorno alto. (En la simulación, el VAN en el percentil 90 es US\$ 1.565 millones. Ello implica que la probabilidad de que el VAN supere los US\$ 1.565 millones es 0,1 (o 10\%).)

Desgraciadamente, estos resultados son dicotómicos, pues dependen de si se impone o no un régimen de control de emisiones de $\mathrm{CO}_{2}$. Aun cuando esto resalta la conveniencia de esperar, no indica si conviene realizar los preparativos necesarios para construir la primera central nuclear 5 .

${ }^{5}$ Es conveniente notar que aun si no se impone un impuesto a las emisiones, existe una externalidad asociada a la generación adicional de gases de efecto invernadero, las que terminan por ocasionar el cambio climático. Por eso la sociedad está pagando un "impuesto" equivalente al daño marginal causado por una tonelada extra de $\mathrm{CO}_{2}$; véase Tol, 2005. 
FIGURA N ${ }^{\circ}$ 4: $\quad$ VALOR ACTUAL NETO DE LA ENERGÍA NUCLEAR, ACTUALIZADO AL 2008 SIN $\mathrm{CO}_{2} \mathrm{ST}>0$

(En dólares de 2005)

VAN en 2008 sin impuesto

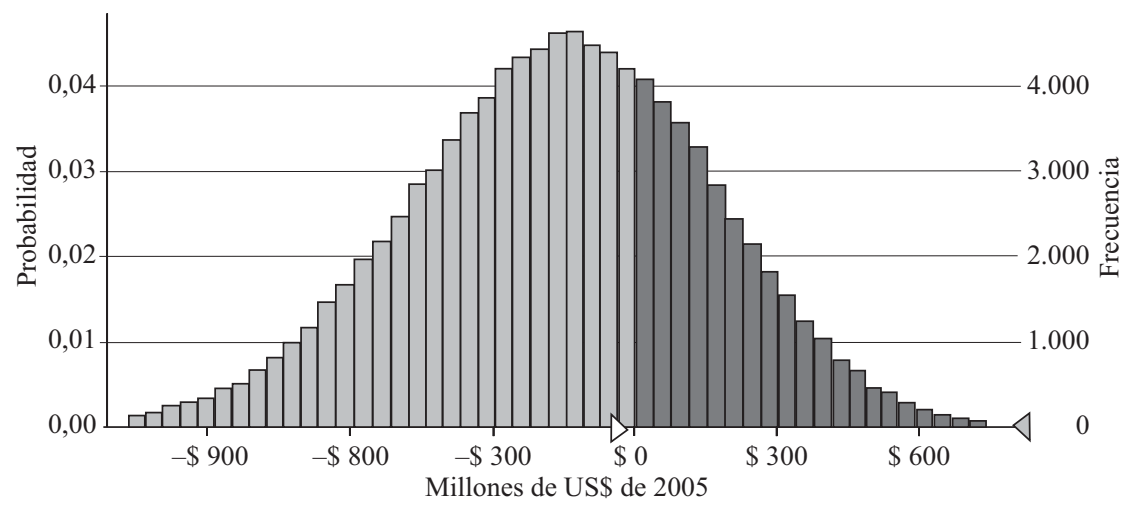

FIGURA N ${ }^{\circ}$ 5: $\quad$ VALOR ACTUAL NETO DE LA ENERGÍA NUCLEAR, ACTUALIZADO AL $2008 \mathrm{CON} \mathrm{CO}_{2} \mathrm{ST}>0$

(En dólares de 2005)

VAN en 2008 con impuesto

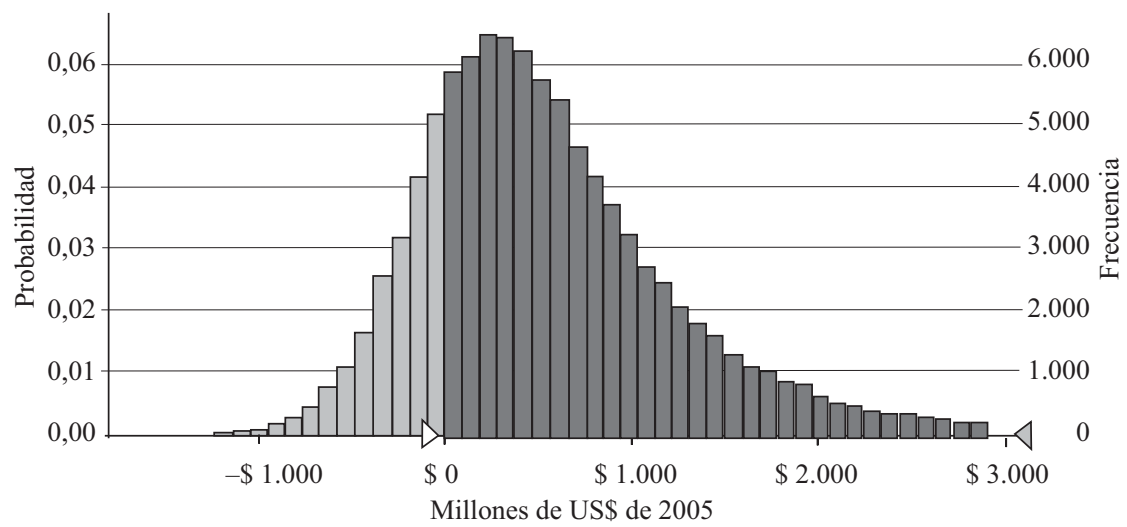

Para resolver esta dicotomía, supongamos que hay entre $10 \%$ y $50 \%$ de probabilidades de que se imponga un $\mathrm{CO}_{2} \mathrm{ST}$ de US\$ 0 a US\$ 50 a los productores de $\mathrm{CO}_{2}$. Ello conduce a la situación observada en la Figura $\mathrm{N}^{\circ}$ 6, donde la probabilidad de un VAN positivo es 0,57 (o 57\%); la mediana es US\$ 66 millones, y la media es US\$ 64 millones, esto es, alrededor de US\$ 65 millones. Así, según este análisis Chile debería estar dispuesto a 
FIGURA N ${ }^{\circ}$ 6: $\quad$ VALOR ACTUAL NETO DE LA ENERGÍA NUCLEAR, ACTUALIZADO AL 2008 CON UNA PROBABILIDAD DE 10\% A 50\% DE QUE SE APLIQUE UNA MULTA POR EMISIÓN DE $\mathrm{CO}_{2}$

(En dólares de 2005)

VAN en 2008 con impuesto

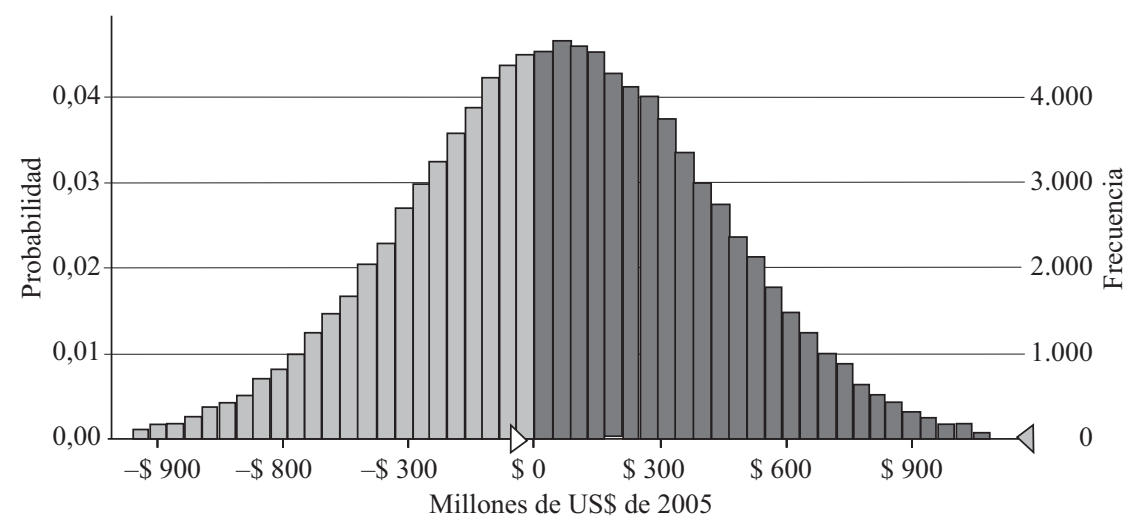

invertir hasta US\$ 65 millones en desarrollar el marco regulatorio necesario para poder optar en 2020 y tomar la decisión de construir una central nuclear 6 .

\section{Conclusión: ¿qué hacer en Chile?}

Teniendo en cuenta los supuestos y los resultados que he presentado, es posible concluir lo siguiente: hasta que se establezca un régimen internacional que obligue a controlar y disminuir las emisiones de gases con efecto invernadero, un país que planee construir su primera central nuclear debería estar dispuesto a invertir un monto positivo (por ejemplo, US\$ 65 millones) en estudios y en desarrollar la institucionalidad regulatoria para tener la opción de construir esa central. Este monto crece si la probabilidad de que se aplique un régimen de control de emisiones de gases de efecto invernadero supera el 50\%. En el resto de esta sección supondré, por simpli-

${ }^{6} \mathrm{~A}$ medida que la probabilidad de que se imponga un régimen de control de emisiones crece hasta 1, el valor presente esperado crece hasta alcanzar US\$ 600 millones. Ésta es sólo una estimación de primer orden del valor de la opción, por lo que es necesario realizar cálculos adicionales empleando un enfoque de opciones reales (véanse Rothwell, 2006, y Graber y Rothwell, 2006). 
cidad, que el valor de la opción de ser capaz de construir una central nuclear en 2020 es de por lo menos US\$ 65 millones a partir de 2008.

Si nos guiamos por el Cuadro $\mathrm{N}^{\circ} 2$, las inversiones en infraestructura física sólo deberían iniciarse cuando exista una institución regulatoria facultada para autorizar la construcción y operación de una central nuclear. Asimismo, suponiendo que en el futuro se conocerá con mayor precisión cuál es la probabilidad de que se aplique un régimen internacional de control de emisiones, deberían destinarse fondos para desarrollar las primeras dos etapas. Una vez que se implemente ese régimen de control de emisiones de carbono, habría que volver a evaluar el proyecto.

Suponiendo que un año de trabajo calificado cuesta US\$ 100.000 (incluyendo beneficios y gastos generales), US\$ 65 millones dan para 650 años-hombre para cumplir con las tareas esbozadas en los primeros tres períodos del Cuadro $\mathrm{N}^{0} 2$. ¿Será posible completar las tareas del primer período con 100 años-hombre (US\$ 10 millones)? ¿Será posible completar las tareas del segundo y el tercer período con 550 años-hombre (US\$ 55 millones), la mitad de los cuales (alrededor de US\$ 24 millones) se dedicaría a la creación de un organismo regulatorio (acreditador) cuya planta incluiría a 30 funcionarios durante ocho años? Chile tendrá que decidir si está dispuesto a gastar estos montos para completar las primeras dos etapas señaladas por la OIEA.

Por último, según la OIEA (2007) la Etapa 1 consiste en “comprometerse, con conocimiento de causa, con un programa de energía nuclear”. Para determinar lo que piensa y quiere la opinión pública se deberán hacer encuestas periódicas. Éstas permitirán evaluar la solidez de los conocimientos y las opiniones de la población respecto de la energía nuclear, y la manera en que las actitudes cambian en el tiempo. En el caso de Chile esa iniciativa costaría menos de un millón de dólares al año.

En consecuencia, la inversión más rentable que Chile podría hacer en este momento sería crear una encuesta longitudinal para evaluar y seguir las actitudes de la opinión pública frente a la energía nuclear. El fin de esta encuesta periódica es cumplir la primera etapa de la OIEA: un compromiso, con conocimiento de causa, con un programa de energía nuclear. (Por cierto que lo anterior no constituye una exigencia sino una recomendación de la OIEA.) Mientras no se asuma ese compromiso, las inversiones en infraestructura física deberían postergarse. 


\section{APÉNDICE \\ Índice de materias de la OIEA (2007)}

\section{Introducción}

1.1. Antecedentes

1.2. Objetivo

1.3. Campo de aplicación

1.4. Usuarios

1.5. Estructura

1.6. Uso de esta guía

2. Programa para desarrollar las etapas

2.1. Etapas de infraestructura

2.2. Etapa 1: Disposición a comprometerse, con conocimiento de causa, con un programa nuclear

2.3. Etapa 2: Disposición a llamar a licitación para la primera central nuclear

2.4. Etapa 3: Disposición a poner en servicio y operar la primera central nuclear

\section{Descripción de aspectos relativos a la institucionalidad regulatoria}

3.1. Posición nacional

3.2. Seguridad nuclear

3.3. Administración

3.4. Aporte de fondos y financiamiento

3.5. Marco legislativo

3.6. Salvaguardias

3.7. Marco regulador

3.8. Protección contra la radiación

3.9. Red eléctrica

3.10. Desarrollo de recursos humanos

3.11. Participación ciudadana

3.12. Emplazamiento e instalaciones de apoyo

3.13. Protección del ambiente

3.14. Planificación de emergencia

3.15. Seguridad y protección física

3.16. Ciclo del combustible nuclear

3.17. Desechos radioactivos

3.18. Participación de la industria

3.19. Adquisiciones 


\section{GLOSARIO}

AL

ALWR

Asia or.

BTU

BWR

CANDU

CCGT

$\mathrm{CO}_{2} \mathrm{ST}$

$\mathrm{D}_{2} \mathrm{O}$

DOE

EIA

Eur. occ.

EUS/EO/AC

GB/CW

GCR

GJ

HWR

IDC

K

kWe

LMR

LWGR

LWR

MOX

MW

MWh

América Latina. Incluye a Argentina, Brasil y México.

(Advanced light water reactors). Reactores avanzados de agua ligera.

Asia oriental. Incluye China, Japón, Corea del Sur y Taiwán.

(British thermal unit) Medida de energía, equivalente al 94,8\% de un gigajoule.

(Boiling water reactor) Reactor de agua en ebullición. La turbina es impulsada por el agua en ebullición del reactor.

(CANadian deuterium-uranium reactor) Reactor atenuado y refrigerado con agua pesada a presión.

(Combined-cycle gas turbine) Turbina impulsada por un ciclo combinado avanzado de gas/petróleo.

El costo de emititir una tonelada de $\mathrm{CO}_{2}$, ya sea a modo de impuesto o de permiso.

Dos átomos de deuterio con un átomo de oxígeno constituyen el agua pesada, que se utiliza como atenuador en los reactores de agua pesada.

(US Department of Energy) Departamento de Energía de los Estados Unidos.

(US Energy Information Administration) Organismo que forma parte del DOE (Departamento de Energía) de Estados Unidos.

Europa occidental. Incluye a Bélgica, Finlandia, Alemania, Italia, Holanda, Eslovenia, España, Suecia y Suiza.

Ex Unión Soviética / Europa oriental / Asia central. Incluye a Armenia, Bulgaria, la República Checa, Alemania Oriental, Hungría, Irán, Rumania, Eslovaquia, Kazajstán, Lituania, Rusia y Ucrania.

Gran Bretaña y el Commonwealth británico. Incluye a Gran Bretaña, Canadá, India, Pakistán y Sudáfrica.

(Gas-cooled reactor) Reactor refrigerado con gas.

Giga-joule.

(Heavy water reactor) Reactor de agua pesada.

(Interés durante la construcción) Costos de financiamiento durante la construcción.

El costo total de la inversión de capital.

Kilowatt eléctrico, medida utilizada para comparar costos entre diversas alternativas.

(Liquid metal reactor) Reactor de metal líquido.

(Light water cooled reactor with a graphite moderator) Reactor refrigerado con agua ligera y con atenuador de grafito.

(Light water reactor) Reactor de agua ligera, el reactor comercial más usado.

Mezcla de óxido de uranio $\left(\mathrm{UO}_{2}\right)$ y óxido de plutonio.

Megawatt (eléctrico). MWth es megawatt térmico.

Megawatt-hora, energía generada por un megawatt de potencia durante una hora.

NCR (US Nuclear Regulatory Commission) Comisión Reguladora de la Energía Nuclear de los Estados Unidos. 
NEA (Nuclear Energy Agency) Agencia para la Energía Nuclear, organismo que forma parte de la OCDE.

O\&M Operación y mantenimiento, por lo general en el contexto de "costo de O\&M".

OIEA Organismo Internacional de Energía Atómica (Viena, Austria).

PWR

$\mathrm{U}$

$\mathrm{U}-235$

$\mathrm{U}-238$

$\mathrm{UF}_{6}$

VAN Valor presente (o actual) neto. Equivale a la suma de todos los flujos de fondos, actualizada a una fecha determinada.

WWER PWR desarrollado en la ex Unión Soviética y en la actual Rusia.

\section{BIBLIOGRAFÍA}

Cabrera-Palmer, B. y G. Rothwell (2008): “Why is Brazil Enriching Uranium?”. En Energy Policy, 36: 2570-2577.

Chupka, M. W. y G. Basheda (2007): "Rising Utility Construction Costs: Sources and Impacts". The Edison Foundation (septiembre).

Economic Modeling Working Group (EMWG) (2007): Cost Estimating Guidelines for Generation IV Nuclear Energy Systems. EMWG of the Generation IV International Forum (GIF).

Energy Information Administration (EIA) (2007): Annual Energy Outlook-2007. U.S. Department of Energy. DOE/EIA-0383.

Graber, R. y G. Rothwell (2006): "Valuation and Optionality of Large Energy Industry Capital Investments". En Cost Engineering, 48, 8: 20-26.

Lapuerta, C. y T. Brown (2007): "Financial Risk and the Relative Economies of Natural Gas, Coal-Fired and Nuclear Power Stations”. Presentado ante el London Energy Forum, London Business School, The Royal Society (oct. 23-25).

Nordhaus, W. D. (2001): “After Kyoto: Alternative Mechanisms to Control Global Warming”. Presentado durante la Reunión Annual de la American Economic Association (4 de enero).

Nuclear Energy Agency (NEA) (2005): Projected Costs of Generating Electricity: 2005 Update. París, Francia: Agencia Internacional de la Energía, Organización para la Cooperación y el Desarrollo Económico.

Organismo Internacional de Energía Atómica (OIEA) (1998): Choosing the Nuclear Power Option: Factors to be Considered. Vienna, Austria: OIEA.

- (2002): Power Reactor Information System (PRIS) Database. Viena, Austria: OIEA.

(2007): Milestones in the Development of a National Infrastructure for Nuclear Power. Viena, Austria: OIEA. http://www-pub.iaea.org/MTCD/publications/PDF/ Pub1305_web.pdf. 
Ray, R. (2008): “Nuclear Costs Explode”. En Tampa Tribune, 15 de enero. http://news.tradingcharts.com/futures/9/7/103360779.html.

Roques, F., W. Nuttall, D. Newberry, R. de Neufville y S. Connors (2006): "Nuclear Power: A Hedge against Uncertain Gas and Carbon Prices?”. En The Energy Journal, $27,4$.

Rothwell, G. (2006): “A Real Options Approach to Evaluating New Nuclear Power Plants”. En The Energy Journal, 27, 1.

Tol, R. (2005): “The Marginal Damage Costs of Carbon Dioxide Emissions: An Assessment of the Uncertainties”. En Energy Policy, 33: 2064-2074.

Vergara, J. (2007): “Energía Nucleo-eléctrica en Chile”. Presentado en la P. Universidad Católica de Chile, Valparaíso, Chile (24 de agosto). 